\title{
Surfactant and Hydrocarbon Aggregates on Defective Graphite Surface: Structure and Dynamics
}

\author{
Maria Sammalkorpi, *, Athanassios Z. Panagiotopoulos, ${ }^{\ddagger}, \S$ and Mikko Haataja ${ }^{\dagger, \S}$ \\ Department of Mechanical and Aerospace Engineering, Department of Chemical Engineering, and Princeton \\ Institute for the Science and Technology of Materials (PRISM), Princeton University, \\ Princeton, New Jersey 08544
}

Received: May 18, 2008; Revised Manuscript Received: August 6, 2008

\begin{abstract}
We have simulated the structure and aggregation kinetics of sodium dodecyl sulfate (SDS) and dodecane $\left(\mathrm{C}_{12}\right)$ on a graphite surface in the presence of point and line defects. We find that while vacancies do not affect the orientational bias of the molecules, they interfere with aggregate formation. Specifically, they disrupt the formation of extended aggregates. Line defects in the form of surface steps, on the other hand, tend to localize the aggregates in their vicinity and induce specific orientations along the step edges. We demonstrate that this orientational bias can be tuned by manipulating the terrace widths. These results suggest that extended defects could be employed to localize and orient surfactant aggregates on the basal plane of graphite, thus providing a means to create patterned aggregate domains.
\end{abstract}

\section{Introduction}

Surfactant adsorption at solid-liquid or liquid-liquid interfaces is key in many industrial processes, including corrosion inhibition, dispersion stabilization, detergency, crude oil refining, purification, and lubrication. Understanding self-aggregation and the physical properties of the resulting aggregate structures on surfaces is often key in further improving these important industrial processes, as well as providing fundamental physical insight into general self-assembly processes. Often, an important aspect of surface aggregation is overlooked: a surface is rarely, if ever, smooth and defect-free, and the presence of irregularities is expected to influence the aggregation process. Here, we continue our studies of sodium dodecyl sulfate (SDS) and hydrocarbon aggregation on an ideal graphite surface ${ }^{1}$ by focusing on the effects of surface defects (vacancies and steps) on the aggregation kinetics of SDS and dodecane $\left(\mathrm{C}_{12}\right)$ monolayers at varying coverages on graphite.

The current state of research in physisorbed layers on surfaces has been reviewed in ref 2. Indeed, surfactant adsorption and the resulting surface structures have been studied by various experimental techniques such as the streaming potential method, ${ }^{3}$ calorimetry, ${ }^{4}$ neutron reflection, ${ }^{5-7}$ ellipsometry ${ }^{8-10}$ and fluorescence spectroscopy, ${ }^{11,12}$ and atomic force microscopy (AFM).${ }^{13-21}$ Of these, AFM provides the most detailed information on the topology of the assembled surfactant structures at molecular scales: Manne et al. first provided evidence of hexadecyltrimethylammonium bromide $\left(\mathrm{C}_{16} \mathrm{TAB}\right)$ organization in parallel stripes at the interface between graphite and aqueous solution. ${ }^{17}$ In subsequent studies, similar morphologies have been observed for various other surfactants and hydrophobic surfaces, ${ }^{13,18,20}$ among them sodium dodecyl sulfate (SDS) on graphite..$^{14,22}$ As the observed stripe spacing was approximately twice the linear size of the surfactant, it has been postulated that a monolayer where the surfactants were placed head-to-

\footnotetext{
* To whom correspondence should be addressed. E-mail: msammalk@ princeton.edu formerly M. Huhtala.

$\dagger$ Department of Mechanical and Aerospace Engineering.

¥Department of Chemical Engineering.

$\S$ Princeton Institute for the Science and Technology of Materials.
}

head acted as a precursor for the formation of hemimicelles. Indications of the formation of such monolayer have been recently observed ${ }^{23,24}$ Much less addressed is the influence of an imperfect or rough surface on the aggregates; refs $25-27$ provide low-resolution indications that surface roughness affects the hemimicelle form and orientation, whereas refs 28 and 29 report the influence of surface roughness on the adsorbed surfactant film structure. Very recently, Schniepp et al ${ }^{30}$ were able to obtain high-resolution images of SDS aggregates on a rough gold surface and report that the hemimicelle morphology (extended vs shorter or irregular-shaped) depends on the local curvature of the surface. Also very recently, Hlawacek et al. published a study on the growth of thin films of a rod-like molecule, para-sexiphenyl, in which the effects of step edges were explored. ${ }^{31}$

From a theoretical/computational perspective, studies of aggregate formation on surfaces to-date typically involve ideal surfaces. Coarse-grained Monte Carlo studies ${ }^{32,33}$ have provided useful information about morphological transitions in surfactant surface aggregates, while molecular dynamics simulations (both coarse-grained and atomistic) ${ }^{34-38}$ have been carried out to investigate aggregate properties at the molecular scale. The initial monolayer and its dynamics on an ideal graphite surface have been studied in ref 1 , wherein it was shown that the graphite surface imposes an orientational bias for both $\mathrm{C}_{12}$ and SDS at the single-molecule level while the bias disappears at intermediate coverage due to intermolecular interactions, only to re-emerge at complete filling. On the other hand, simulations of aggregate dynamics on defective surfaces are quite rare. For example, diffusion of alkene molecules on stepped surfaces was studied in ref 39 , while ref 40 reported on hydrocarbon behavior on chiral platinum surfaces. Finally, $\mathrm{O}_{2}$ adsorption and desorption on stepped gold surfaces was studied in ref 41 .

To our knowledge, the effects of surface defects on the aggregation kinetics of surfactants on graphite have not been addressed computationally. In this work, we provide a microscopic picture of how defects affect initial aggregation of SDS and $\mathrm{C}_{12}$ on a graphite surface through molecular dynamics simulations. Our results show that point defects (vacancies) 
affect the stability of the aggregates without affecting surfactant orientations. On the other hand, extended line defects (surface steps) impose orientational order for surfactants, which may possibly be employed to orient and localize surfactant aggregates.

The rest of this article is organized as follows. The computational model is detailed in section 2, while section 3 contains the description of the results. Finally, section 4 is dedicated to a discussion of the results.

\section{Methods}

2.1. Computational Model. The Gromacs 3.3 simulation package ${ }^{42-44}$ including Lennard-Jones and Coulombic site-site interactions in addition to bond stretching, angular bending, and improper and proper dihedral interactions with the united atom parametrization ${ }^{45}$ was employed for the molecular dynamics (MD) simulations. After the simulations were initialized by minimizing the energies of the initial configurations with the steepest descent method, all simulations were performed in the NVT ensemble using the Berendsen thermostat ${ }^{46}$ with a coupling time constant of $0.1 \mathrm{ps}$. SDS/ $\mathrm{C}_{12}$ molecules, sodium counterions, and the water molecules were thermostatted as separate groups with the temperature maintained at $T=323 \mathrm{~K}$. The bond lengths of the detergent and hydrocarbon molecules were constrained by the LINCS algorithm ${ }^{47}$ and those of the water molecules by SETTLE. ${ }^{48}$ A time step of 2 fs was employed in all simulations reported here. The Lennard-Jones interactions were cut off at 1 $\mathrm{nm}$, and the full particle-mesh Ewald method ${ }^{49}$ was employed for the long-ranged electrostatic interactions in order to minimize artifacts resulting from artificially truncating such interactions. ${ }^{50-52}$ The Simple Point Charge (SPC) model $^{53}$ was adopted for the water molecules.

The SDS parametrization employed in the work is described in detail in ref 54 and is available online at http://www.apmaths. uwo.ca/ $\sim$ mkarttu/downloads.shtml. The remaining parameters follow the united atom parametrization of ref 45 . The graphite carbon atom is described by the Gromacs carbon atom type CB, which is placed in a hexagonal pattern with $1.39 \AA$ as the nearest-neighbor distance, corresponding to the equilibrium $\mathrm{CB}-\mathrm{CB}$ distance. The atom type $\mathrm{CB}$ was chosen because it represents a bare carbon atom in a five- or six-membered ring, and thus, it was considered to best represent the $\mathrm{sp}^{2}$-hybridized carbon in graphite. For the graphite step, the edge was terminated by $\mathrm{CH}_{2}$ methyl groups with the aim of describing hydrogen termination of the graphite bonds. In the simulations, all atoms describing the graphite were fixed. We also verified that the results were not cut-dependent, that is, cleaving the graphite layer in such a way that $\mathrm{CH}_{3}$ termination is required did not influence the results in comparison to a cut corresponding to $\mathrm{CH}_{2}$ termination with the same orientation. Furthermore, we have previously verified that the results are not stackingdependent, that is, ABA stacking produces the same results as ABC stacking. ${ }^{1}$ A single graphene layer was sufficient to induce the observed orientational behavior at the single-molecule level, but to ensure that molecules adsorbed at the opposing plane would not interact with the molecules on the defective surface, two or three layers were employed in this work. Throughout the simulations, periodic boundary conditions were employed to create an infinite graphite surface or a semi-infinite step. We would like to point out that although long-range electrostatic interactions are fully taken into account in the simulations, the relatively small system size and unavoidable use of periodic boundary conditions affect the formation of spatially extended structures, such as worm-like hemimicelles. Great care must thus be used in drawing conclusions on such extended structures

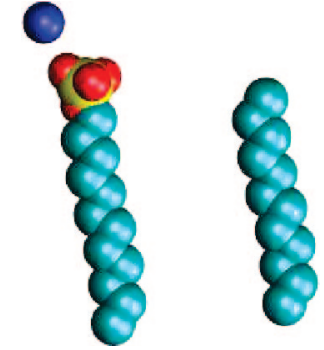

Figure 1. SDS and $\mathrm{C}_{12}$ molecules. Each cyan sphere represents a $\mathrm{CH}_{2}$ or a $\mathrm{CH}_{3}$ group.

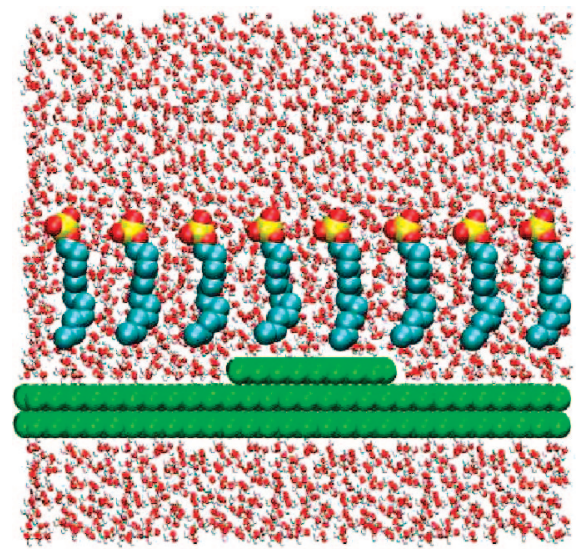

Figure 2. An example of the initial configuration, here 64 SDS molecules on a stepped graphite surface.
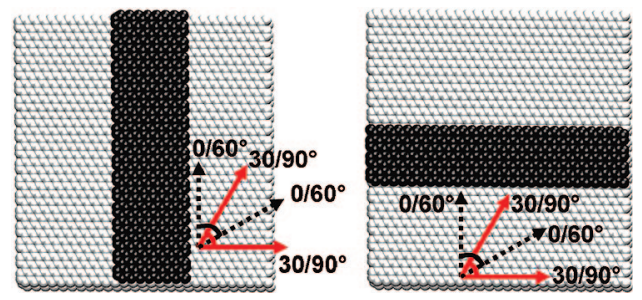

Figure 3. The two step orientations examined in this work. The image on the left corresponds to a step with lattice cleavage along one of the vectors corresponding to a $0 / 60^{\circ}$ angle with respect to graphite nearestneighbor bond, and the image on the right corresponds to cleavage in a $30 / 90^{\circ}$ angle with respect to graphite bonds. The former (latter) orientation is referred to as " $\mathrm{A}$ " (" $\mathrm{B}$ ") in the analysis below.

based on the simulation results. Visualizations of all molecular configurations were done using VMD. ${ }^{55}$

2.2. Simulated System. For all of the initial configurations, three graphene planes are initially stacked in ABC stacking with an interlayer distance of $3.35 \AA$ A. Next, from 1 to 64 SDS or $\mathrm{C}_{12}$ molecules (ideal and vacancy surface) or 9 and 64 SDS/ $\mathrm{C}_{12}$ molecules (stepped surface) are placed above the graphite surface with their axis perpendicular to the graphite plane (see Figures 1 and 2), and the simulation box is filled with water molecules using the Gromacs genbox algorithm with default van der Waals distance $0.105 \mathrm{~nm}$, after which the system is minimized in energy and then simulated for 50 (ideal systems and systems with vacancies) or $100 \mathrm{~ns}$ (systems with steps). The steps bind the molecules strongly, and thus, the longer trajectories provide an improved sampling of the phase space.

For an ideal graphite surface, three graphite layers are fixed in perfect $\mathrm{ABC}$ stacking. In the case of vacancies, on the other hand, the topmost graphite layer is embedded with 12 equally spaced vacancies (see Figure 4 for the defect configuration), while the two remaining layers remain ideal. 


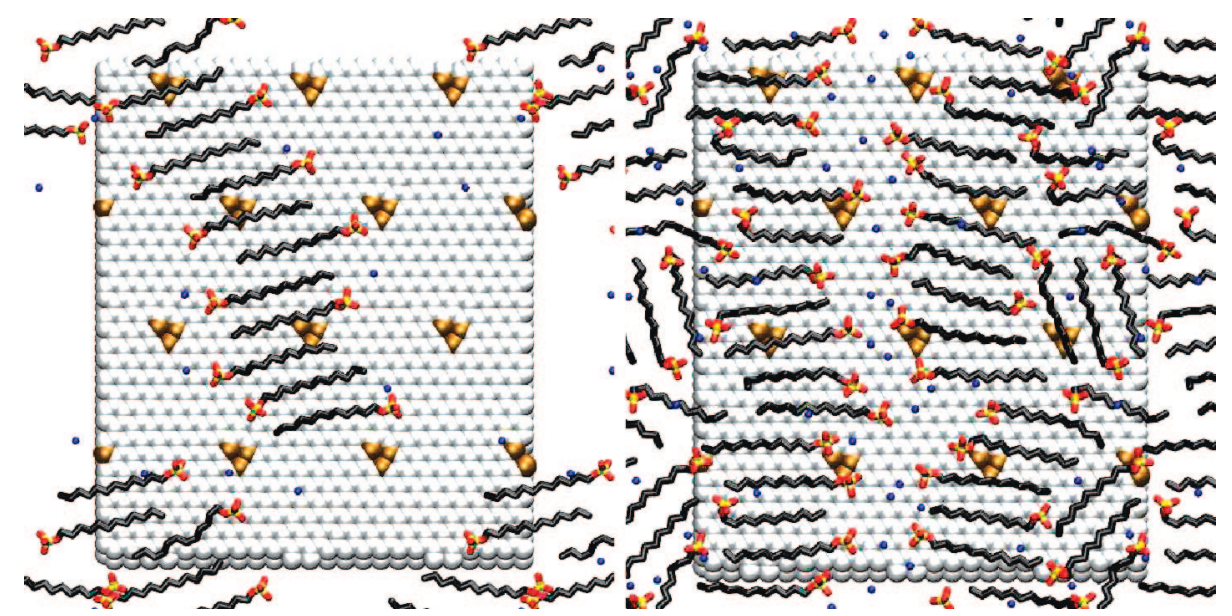

Figure 4. Snapshots of SDS configurations with 16 SDS (left) and 49 SDS (right) systems at 50 ns on a surface with vacancies. The vacancy sites are highlighted in the image. Note that a small portion of the molecules remain in the bulk water and are not shown on the surface visualization. These molecules are also disregarded in the analysis.

On the basis of density functional theory (DFT) calculations, it is well-known that on planar graphite, a relaxed monovacancy configuration has two of the dangling bonds partially reconstructing and the third bond emerging out of the plane. ${ }^{56-58}$ Whereas non-spin-polarized DFT calculations predict this protrusion to be larger, ${ }^{56,57}$ DFT calculations taking into account spin polarization predict an out-of-plane protrusion of $0.18 \AA .^{58}$

As DFT or advanced tight binding calculations are required to reproduce the monovacancy relaxation accurately, we resorted to exporting a DFT relaxed vacancy configuration to the Gromacs simulations. The DFT-based relaxation was performed in a unit cell of 127 atoms using the plane wave basis Vienna ab initio simulation pack (VASP) code ${ }^{59,60}$ and implementing the spin-polarized DFT with the generalized gradient approximation; for more details on relaxation process, see ref 58 . To make the 127 atom unit cell containing a single relaxed monovacancy compatible with the Gromacs simulation, the atom coordinates were scaled in the direction of the graphite plane so that interatomic distance in defect-free regions was the Gromacs $\mathrm{CB}-\mathrm{CB}$ distance of $1.39 \AA$ instead of the DFTpredicted $1.42 \AA$, after which the unit cell was copied 12 times to produce the 12 vacancy fixed graphite layer shown in Figure 4. The emerging dangling bond corresponding to each vacancy resides $0.18 \AA$ out-of-plane. ${ }^{58}$ Although the scaling of the bond lengths makes the vacancy geometry less accurate, it is necessary to maintain self-consistency within the simulation as the Gromacs $\mathrm{CB}-\mathrm{CB}$ distance differs slightly from the DFTpredicted graphite interbond distance.

In the case of line defects, that is, steps, on graphite, a rectangular portion of the topmost layer was first removed to create a step. Two different orientations of the cut were studied, one corresponding to a $0 / 60^{\circ}$ angle on the graphite plane and the other to the $30^{\circ}$ angle; see Figure 3 . For each orientation, two different step widths were produced, (1) a wide step (effectively a trench) and (2) a narrow step. Next, the atoms at step edges were replaced by methyl groups to describe hydrogen termination of the dangling bonds. We note that this is a crude approximation of the edge; the atoms are fixed, that is, no relaxation is allowed at the edge, and the model does not allow for reactivity. In our judgment, this approximation is reasonable for exploring the effects of the geometry of the step edges on aggregate structure and dynamics.

For the ideal graphite surface and the step configurations, a graphite slab of size $6.74 \mathrm{~nm} \times 7.09 \mathrm{~nm}$ was employed (1904 carbon atoms in each full layer) for all of the multiparticle simulations reported here, while a graphite slab of size 4.57 $\mathrm{nm} \times 5.00 \mathrm{~nm}$ (912 carbon atoms in each layer) was employed for single $\mathrm{SDS} / \mathrm{C}_{12}$ simulations. The total thickness of the simulation box (i.e., the combined thickness of the graphite and the water slab) was set to $z=4.5 \mathrm{~nm}$ for the simulations involving a single $\mathrm{SDS} / \mathrm{C}_{12}$ molecule and $z=8.5 \mathrm{~nm}$ for multiparticle simulations. In the case of ideal graphite, this corresponds to 2533 water molecules (SDS) or 2540 water molecules $\left(\mathrm{C}_{12}\right)$ in the smaller simulation box and 10739-11761 water molecules in the larger box, depending on the number of $\mathrm{SDS}$ or $\mathrm{C}_{12}$ molecules. For the graphite systems with the step, a smaller water region thickness $(z=6.5 \mathrm{~nm})$ was found to be sufficient; this corresponds to 7591-8835 solvent molecules.

A graphite slab with 12 vacancies within the topmost layer was chosen to have dimensions of $5.78 \mathrm{~nm} \times 6.67 \mathrm{~nm}$, which contains $1524+1536+1536=4596$ graphite atoms in total in the three layers. Physically, these choices correspond to a vacancy areal density of $3.11 \times 10^{17} / \mathrm{m}^{2}$. The thickness of the simulation box was set to $8.5 \mathrm{~nm}$, and the simulated systems contained 8142-9153 water molecules, depending on the number of $\mathrm{SDS} \mathrm{C}_{12}$ molecules.

Finally, we note that only a finite fraction of the molecules end up on the surface during the course of the simulation. To ensure that the surface aggregates were not directly influenced by the molecules in the bulk, sufficiently large simulation boxes were employed in the direction perpendicular to the surface plane. We explicitly verified that this was the case for all of the cases studied here. For the systems with the ideal surface and the surface with vacancies, surfactant orientation results from a delicate balance between various forces, ${ }^{1}$ thus requiring a water slab thickness of $8.5 \mathrm{~nm}$ for multiple molecule simulations. For the surface with a step, the influence of the step was found to be dominant, so that a $6.5 \mathrm{~nm}$ slab thickness was observed to be more than sufficient.

\section{Results}

The following simulation protocol was employed. Starting from an initial configuration, similar to the one shown in Figure 2 , simulations show that the molecules either rapidly migrate to the surface or form small aggregates in bulk solvent within the first few nanoseconds. Aggregates remaining in bulk solvent typically contain up to $15 \%$ of the $\mathrm{SDS} / \mathrm{C}_{12}$ molecules in the system, depending on the simulation trajectory. The first $10 \mathrm{~ns}$ 


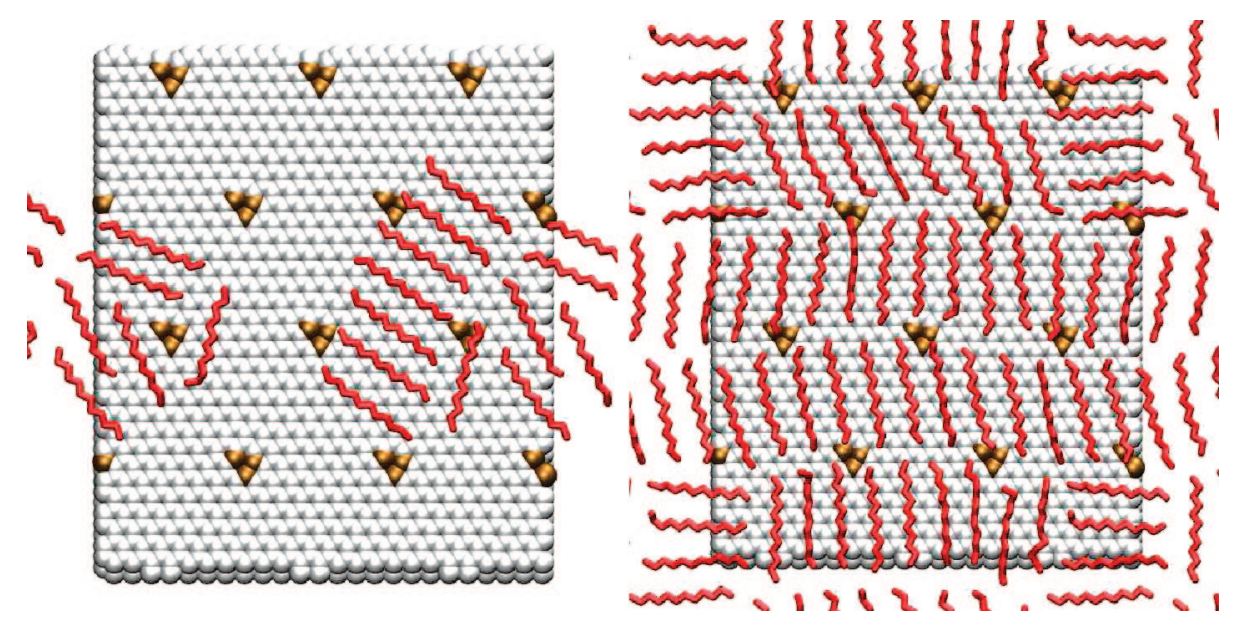

Figure 5. Snapshots of $C_{12}$ configurations with $16 C_{12}$ (left) and $64 C_{12}$ (right) systems at $50 \mathrm{~ns}$ on a surface with vacancies. The vacancy sites are highlighted in the image. Note that a small portion of the molecules remains in the bulk water, and they are not shown on the surface visualization. These molecules are also disregarded in the analysis.

of all simulations were thus considered as the initial relaxation period and were not included in the data analysis. The remainder of the 50 (for the ideal surface or the surface with vacancies) or $100 \mathrm{~ns}$ (for the stepped surface) trajectory formed the basis for the analysis of the results reported here. We note, however, that the time scales accessible by direct simulations currently are insufficient to describe equilibration between adsorption and desorption from and to the bulk. Therefore, in the subsequent analysis and discussion, only the molecules on the surface are considered. While global equilibrium cannot be attained in the current simulations, we believe that our data captures the local influence of surface defects on aggregation kinetics and structure; ref 1 covers questions related to the $\mathrm{SDS} / \mathrm{C}_{12}$ monolayer behavior on ideal surface, whereas ref 54 provides an extensive discussion of SDS micellization in the bulk through molecular dynamics simulations.

3.1. Vacancies. From the perspective of molecular orientations on the surface, vacancies did not have an observable effect compared to the pristine surface. On the other hand, vacancies do affect the aggregate dynamics, especially in the case of $\mathrm{C}_{12}$. To this end, partially and fully filled final, that is, $50 \mathrm{~ns}$, configurations of SDS and $\mathrm{C}_{12}$ monolayers on a surface with vacancies are presented in Figures 4 and 5, respectively. For SDS, very little difference between the ideal and defective surface is observed visually, while visual analysis of $\mathrm{C}_{12}$ trajectories indicates that the aggregates are less ordered and less compact in the presence of vacancies at submonolayer coverage. For filled monolayers, on the other hand, visual inspection does not provide a significant difference between the ideal and defective surface.

To quantify these observations, we calculated the size distribution of the monolayer-thick mobile islands that are formed at submonolayer coverages. Here, an island is defined on the basis of the following geometrical rule: A molecule is part of an island if three or more of its methyl groups are closer than $R_{\text {cut }}=0.45 \mathrm{~nm}$ to three methyl groups in a different SDS/ $\mathrm{C}_{12}$ molecule. The neighboring groups can be in the same molecule (side-by-side orientation) or in different molecules (perpendicular orientation to a side-by-side set). This method of defining an island together with the choice of $R_{\text {cut }}=0.45$ nm was observed to agree well with visual inspection of the trajectories. The analysis shows that SDS islands are much more loosely bound than $\mathrm{C}_{12}$ islands, regardless of the presence or absence of surface defects. Furthermore, the islands are less stable in the presence of vacancies both for SDS and, more
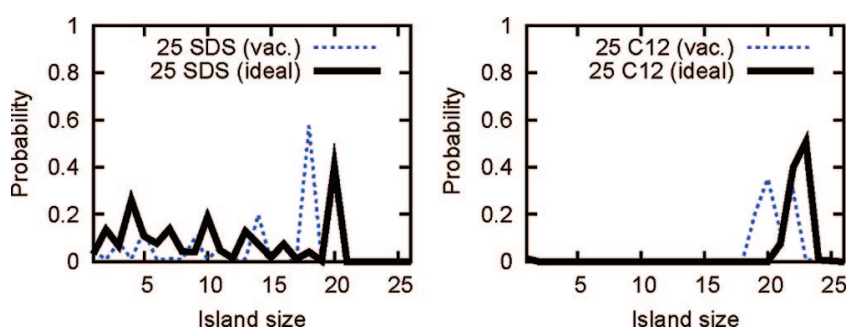

Figure 6. Island size on defective and defect-free surfaces for SDS and $\mathrm{C}_{12}$ at submonolayer coverages. For $\mathrm{C}_{12}$, the vacancies interfere with the aggregation process leading to smaller islands. A similar trend is observed for SDS, for which the islands are less strongly bound to begin with.

prominently, for $\mathrm{C}_{12}$; see Figure 6 . The decreased stability is reflected in a smaller most-probable island size and broader overall size distribution. The more loosely bound SDS islands result from the head-head repulsion with SDS, whereas the headhead repulsion dominates in disrupting the SDS aggregates over the effect of the vacancies, thus providing disrupted aggregates already for a pristine surface; with $\mathrm{C}_{12}$, there is no such intrinsic aggregate disruptive factor. Interestingly, evaluation of the gauche defect probabilities reveals that molecular conformations within the islands are relatively insensitive to the presence of vacancies. This indicates that the effect of the vacancies is not strong enough to affect the conformational equilibrium of the molecules.

Finally, we note that the spatial distributions of the methyl and $\mathrm{SO}_{4}$ groups are affected by the vacancies. Specifically, at low coverages, the adsorbed molecules tend to avoid the vacancies, while increased overlap with the vacancies occurs at elevated coverage, as shown in Figure 7. This is enforced by the coverage pushing individual molecules on the vacancies; collectively, the linear molecular aggregates, especially those consisting of $\mathrm{C}_{12}$ molecules, still tend to avoid vacancies; see Figures 4 and 5 .

3.2. Steps. While vacancies were found to have a relatively small effect on aggregate dynamics, extended defects have the capability of altering the large-scale behavior of the system. The interesting question specific to the case under scrutiny is whether the steps can induce preferred orientations and spatial localization of the molecules, and the answer is affirmative. First, a visual analysis of the trajectories reveals that the steps affect strongly both molecular positions and orientations. More precisely, the molecules are attracted by the rising step edges, 

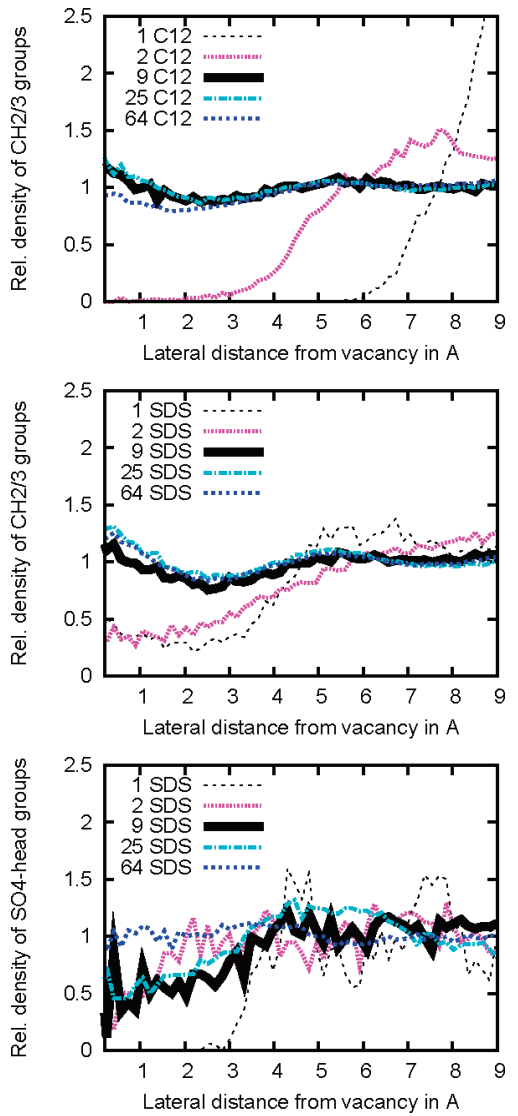

Figure 7. Relative density of $\mathrm{CH}_{2 / 3}$ and $\mathrm{SO}_{4}$ groups as a function of lateral distance to a vacancy. The density is scaled so that in the case of uniform coverage, it equals 1, and any deviation from one is the proportional difference in the observed density.

and once they are located there, they tend to slide along the edges. The attraction of the molecules to the steps is due to the van der Waals interactions between the adsorbed molecules and the surface. On the other hand, molecules which reside on top of the step tend to either avoid the step edges or, if the top of the step becomes filled, prefer orientations in which the molecule head/ end is next to the dropping edge and the rest of the molecule resides in the interior of the step. The behavior is similar for both SDS and $\mathrm{C}_{12}$, although SDS configurations show more disorder due to the head-head repulsion. Figures 8 and 9 present examples which demonstrate this behavior. Again, the behavior results from van der Waals interactions; regions close to the edge are unfavorable because the surface drops at the edge, thus reducing surface contact for molecules close to the edge. Interestingly, these observations imply that the width of the step can be employed to impart a preferred orientation on top of the step, given that the step width is smaller than the linear dimension of the surfactant.

To quantify these observations, we calculated the orientation distribution of the adsorbed molecules both in the upper and lower planes of the stepped graphite surface; see Figure 10. These graphs show that, indeed, a step orients molecules next to it along the step edge, whereas molecules on top of the step span the step with an appropriate orientational tilt to adjust to the step width for relatively narrow steps. For wider steps, the molecules tend to avoid the step edges and attain random orientations, as was previously observed in the case of partially filled monolayers on an ideal surface. ${ }^{1}$ It is plausible that the transition from spanning the step width by adjusting the tilt angle to random orientation may take place through some intermediate

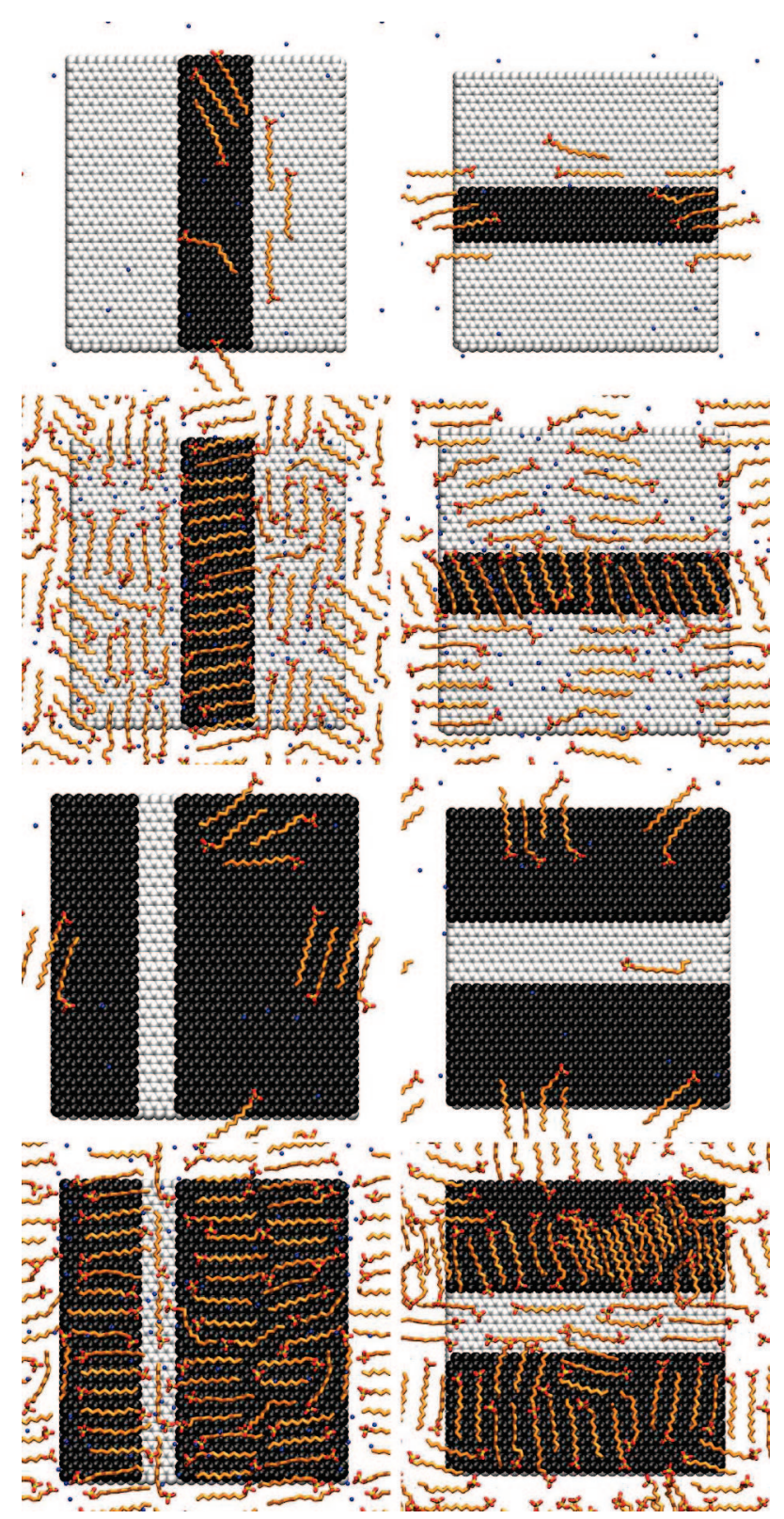

Figure 8. SDS configurations after $100 \mathrm{~ns}$ on a graphite surface with partial and full monolayer coverage on steps of varying width and orientation. The dark graphite layer is on top of the light one. The left column corresponds to orientation A in Figure 3, whereas the right column has step orientation B.

oriented structures such as herringbone or side-by-side orientations for high degrees of filling as the step becomes wider; however, a systematic study of this is not currently feasible due to the high computational cost. The influence of steps is also reflected in the position distribution of the methyl groups with respect to the step edge, as shown in Figure 11. In all cases, a rising step edge attracts molecules strongly and orients them along the step. This orientational effect is carried on further from the step; the spatial oscillations in the methyl group distribution reflect the orientational ordering of nearby hydrocarbon chains. This observation indicates that a step on the surface may be used to enforce an orientation to a monolayer adsorbed on the surface adjacent to the step.

\section{Discussion}

In this communication, we have studied the effects of point and line defects on the $\mathrm{SDS} / \mathrm{C}_{12}$ aggregate structure and kinetics 


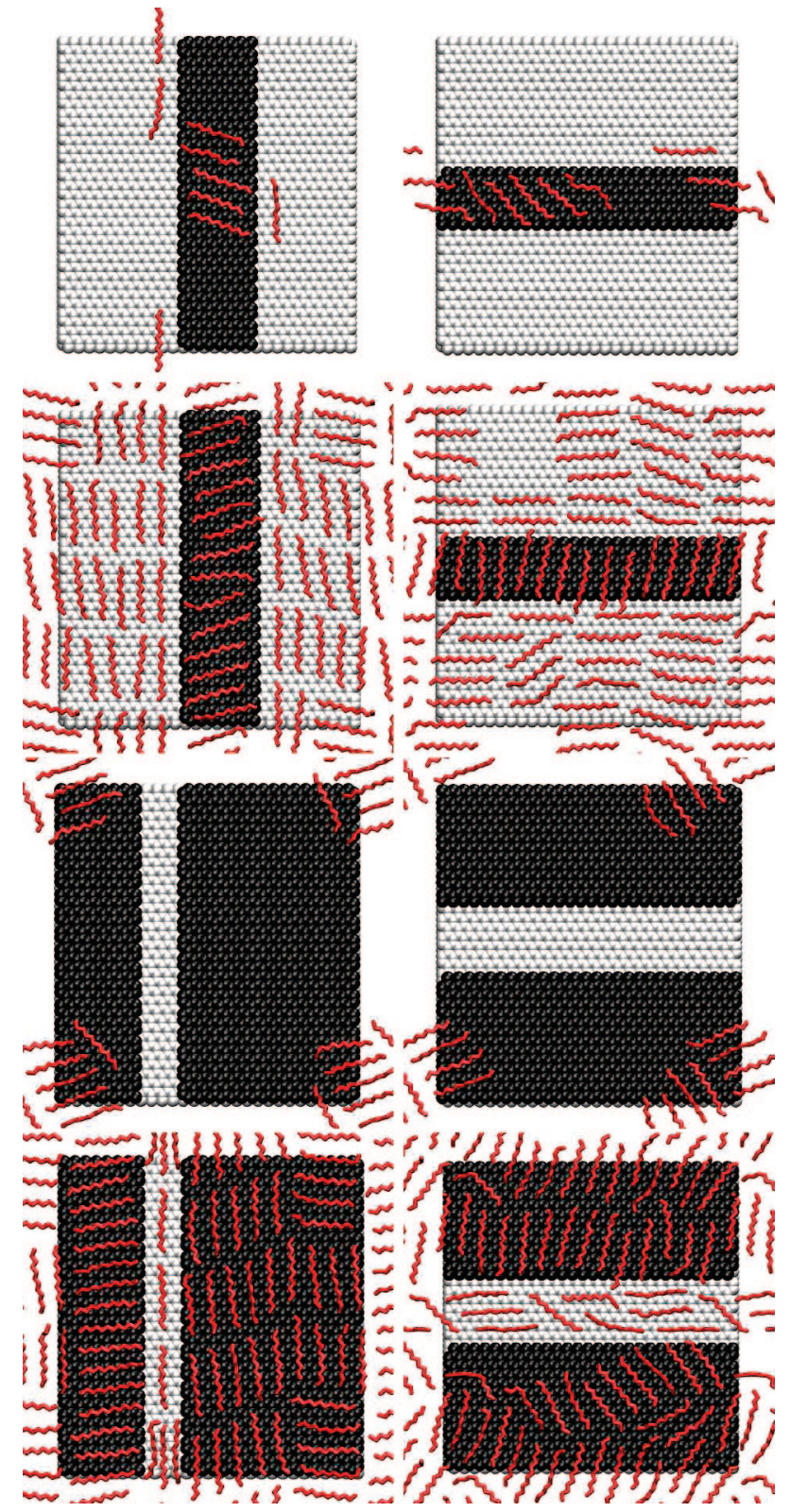

Figure 9. $\mathrm{C}_{12}$ configurations after $100 \mathrm{~ns}$ on a graphite surface with partial and full monolayer coverage on steps of varying width and orientation. The dark graphite layer is on top of the light one. The left column corresponds to orientation A in Figure 3, whereas the right column has step orientation B.

on graphite, specifically their impact on molecular orientation. In the case of vacancies, we have found that while they do not affect the molecular orientation distribution, they interfere with aggregate formation and lead to smaller aggregate sizes at intermediate surface coverage. Furthermore, their influence on aggregate stability is more pronounced for $\mathrm{C}_{12}$ than for SDS because of the presence of the charged head groups in the latter, leading to a weaker attraction to the surface.

These results were obtained within an approach which treats the vacancy as a nonreactive, static entity over the time scale of the simulations (50 ns). This is not quite correct as it is wellknown that a graphite monovacancy at finite temperature is a reactive, dynamic entity. Although vacancy migration in graphite at room temperature is unlikely with migration energies typically reported in the range of a few eV, ${ }^{56,61}$ there are indications that covalent binding can take place through vacancies in graphitebased structures. ${ }^{57,62}$ Our results indicate that the geometric
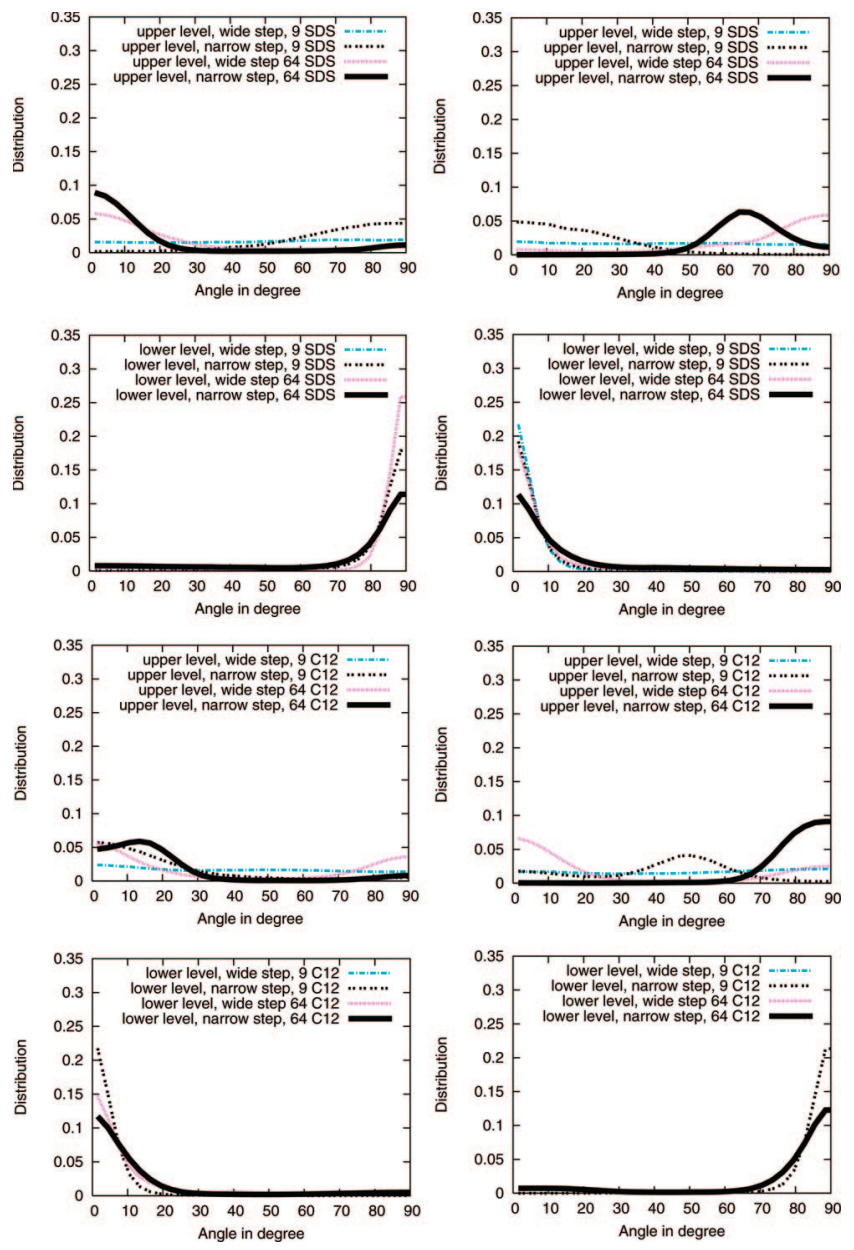

Figure 10. Molecular orientation distributions in systems with a step. The angle is calculated with respect to the horizontal direction of Figure 3. The left column represents step orientation A in Figure 3 and the right column step orientation B. Note that not all systems have molecules both on the lower and upper level, depending on the number of molecules in the system and on the step width.
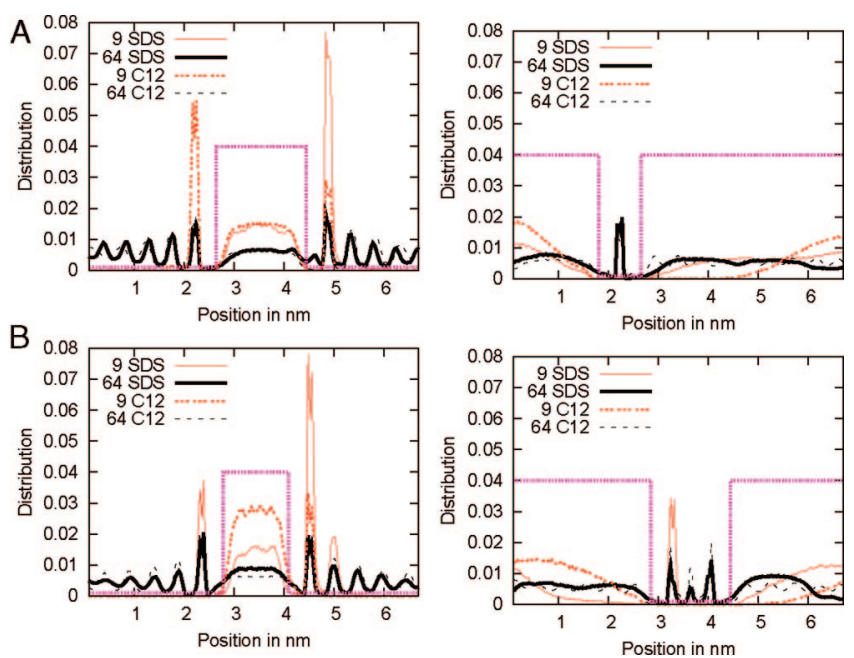

Figure 11. $\mathrm{CH}_{2 / 3}$ distribution as a function of position with respect to the step. The step edges are visualized by the dashed step. The top row represents step orientation A in Figure 3 and the bottom row step orientation B.

effect of vacancies on the surface aggregation of hydrocarbons on graphite is rather small. It is tempting to speculate, however, that covalent bond formation between surfactants and vacancies could be employed to localize surfactant aggregates in selective 
regions with high vacancy density due to, for example, electron irradiation. However, the significance of this should not be overestimated as dangling bonds in defective graphite are likely to be hydrogen-terminated under experimental aqueous conditions, thus reducing the reactivity.

In contrast to point defects, extended defects in the form of step edges were observed to have a very strong effect on molecular orientation and spatial localization. In particular, molecules orient along the rising step edges, and intermolecular interactions impart this preferred orientation to neighboring molecules for both SDS and $\mathrm{C}_{12}$ at elevated coverage. These observations are in qualitative agreement with experimental observations in refs 25-30, wherein micelle morphology was observed to correlate with surface roughness (curvature). The results are in excellent aggreement with the simulations reported in ref 39 , in which alkene molecules were observed to be attracted to the rising steps on a metal surface and to align along the steps. Assuming that the orientation of the initial monolayer governs the formation of the hemimicellar aggregate, our results directly indicate that the presence of steps can be employed to guide the formation and orientation of the micellar aggregate. Furthermore, we have observed indications that the step width can be employed to orient the molecules to an arbitrary orientation on top of the step; this is due to the tendency of the molecules to span a sufficiently narrow step for both SDS and $\mathrm{C}_{12}$. Overall, the orientation due to steps is deduced to result from the hydrocarbon tail, thus allowing for the conclusion that the steps are also likely to orient other surfactant aggregates than SDS and $\mathrm{C}_{12}$. Furthermore, increasing the length of the hydrocarbon chain is expected to amplify this effect.

Again, it is noteworthy that the model employed for the step edges is a drastic simplification in that it is nonreactive, fixed, and hydrogen-terminated. While our results indicate that the geometric effect of step edges is significant and thus can contribute to the orientation and morphology of micelles, it would be interesting to investigate the effect of a reactive step edge on molecular aggregate kinetics and structure. While this is currently not feasible, we expect that improvements in computational capabilities and models will provide means to study these interesting and important systems with improved physical descriptions. However, as noted before, reactivity in an aqueous system should be less of an issue in our opinion, as all sites are likely to be saturated.

Finally, we would like to note that the effect of surface defects on large-scale aggregate structures cannot be readily inferred from such microscopically detailed simulations as they are limited in both spatial and temporal extent. As an alternative computational approach, we are currently developing coarsegrained implicit-solvent models, based on the simulations reported here, which have the capability of accessing aggregate structure and dynamics across mesoscopic length and time scales. This is particularly interesting as defects, more precisely irradiation-induced defects, have been demonstrated as a means to high-precision engineering of the properties of graphitic materials; for a review, see ref 63. For example, recent experimental work has shown that a graphite surface can be patterned either locally via scanning tunneling microscopy ${ }^{64}$ or globally via electron irradiation; ${ }^{65}$ both methods give rise to surface grooves and holes of variable size. Such patterned surfaces should display interesting aggregate structures, and computational approaches would be useful in elucidating the correlation between surface and aggregate structures.

In closing, we would like to note that the results presented in this paper clearly demonstrate that defects on a graphite surface can be employed to localize and orient small surfactant aggregates. These observations provide interesting prospects for guiding and controlling surface self-assembly processes for patterned functional surfaces and templates.

Acknowledgment. The authors thank Dr. Arkady Krasheninnikov for providing the relaxed graphite vacancy configurations. This work has been supported by the Academy of Finland (M.S.), through an NSF-DMR Grant No. 0449184 (M.H.), and by the NSF-MRSEC Program, Grant No. DMR-0213706, at Princeton University.

\section{References and Notes}

(1) Sammalkorpi, M.; Panagiotopoulos, A. Z.; Haataja, M. J. Phys. Chem. B 2008, 112, 2915-2921.

(2) Bruch, L. W.; Diehl, R. D.; Venables, J. A. Rev. Mod. Phys. 2007, $79,1381-1454$.

(3) Fuerstenau, D. W. J. Phys. Chem. 1956, 60, 981-985.

(4) Király, Z.; Findenegg, G. H. J. Phys. Chem. B 1998, 102, 12031211.

(5) McDermott, D. C.; McCarney, J.; Thomas, R. K.; Rennie, A. R. J. Colloid Interface Sci. 1994, 162, 304-310. 1412 .

(6) Penfold, J.; Thomas, R. K. J. Phys.: Condens. Matter 1990, 2, 1369-

(7) Penfold, J.; Staples, E. J.; Tucker, I.; Thompson, L. J. Langmuir 1997, 13, 6638-6643.

(8) Tiberg, F.; Joensson, B.; Tang, J.; Lindman, B. Langmuir 1994, 10, 2294-2300.

(9) Tiberg, F.; Joensson, B.; Lindman, B. Langmuir 1994, 10, 37143722.

(10) Luciani, L.; Denoyel, R. J. Colloid Interface Sci. 1997, 188, 7580.

(11) Levitz, P.; van Damme, H.; Keravis, D. J. Phys. Chem. 1984, 88, $2228-2235$.

(12) Chandara, P.; Somasundaranan, P.; Turro, N. J. J. Colloid Interface Sci. 1987, 117, 31-46.

(13) Manne, S.; Gaub, H. E. Science 1995, 270, 1480-1482.

(14) Wanless, E. J.; Ducker, W. A. J. Phys. Chem. 1996, 100, 32073214.

(15) Ducker, W. A.; Grant, L. M. J. Phys. Chem. 1996, 100, 1150711511 .

(16) Ducker, W. A.; Wanless, E. J. Langmuir 1996, 12, 5915-5920.

(17) Manne, S.; Cleveland, J. P.; Gaub, H. E.; Stucky, G. D.; Hansma,

P. K. Langmuir 1994, 10, 4409-4413.

(18) Patrick, H. N.; Warr, G. G.; Manne, S.; Aksay, I. A. Langmuir 1997, 13, 4349-4356.

(19) Patrick, H.; Warr, G.; Manne, S.; Aksay, I. Langmuir 1999, 15, $1685-1692$.

(20) Jaschke, M.; Butt, H.-J.; Gaub, H. E.; Manne, S. Langmuir 1997, $13,1381-1384$.

(21) Schönherr, H.; Kremer, F. J. B.; Kumar, S.; Rego, J. A.; Wolf, H.; Ringsdorf, H.; Jasche, M.; Butt, H.-J.; Bamberg, E. J. Am. Chem. Soc. 1996, 118, 13051-13057.

(22) Ducker, W. A.; Wanless, E. J. J. Phys. Chem. 1996, 100, 32073214.

(23) Kawasaki, H.; Ban, K.; Maeda, H. J. Phys. Chem. B 2004, 108, 16746-16752.

(24) Király, Z.; Findenegg, G. H. Langmuir 2005, 21, 5047-5054.

(25) Ducker, W.; Grant, L. J. Phys. Chem. 1996, 100, 11507-11511.

(26) Schulz, J.; Warr, G. Langmuir 2002, 18, 3191-3197.

(27) Liu, J.-F.; Ducker, W. J. Phys. Chem. B 1999, 103, 8558-8567.

(28) Fragneto, G.; Thomas, R.; Rennie, A.; Penfold, J. Langmuir 1996, $12,6036-6043$.

(29) Macakova, L.; Blomberg, E.; Claesson, P. Langmuir 2007, 23, $12436-12444$.

(30) Schniepp, H.; Shum, H.; Saville, D.; Aksay, I. J. Phys. Chem. B 2007, 111, 8708-8712.

(31) Hlawacek, G.; Puschnig, P.; Frank, P.; Winkler, A.; AmbroschDraxl, C.; Teichert, C. Science 2008, 321, 108-111.

(32) Wijmans, C.; Linse, P. J. Phys. Chem. 1996, 100, 12583-12591.

(33) Zheng, F.; Zhang, X.; Wang, W.; Dong, W. Langmuir 2006, 22, 11214-11223.

(34) Srinivas, G.; Nielsen, S. O.; Moore, P. B.; Klein, M. L. J. Am. Chem. Soc. 2006, 128, 848-853.

(35) Bandyopadhyay, S.; Shelley, J.; Tarek, M.; Moore, P.; Klein, M. J. Phys. Chem. B 1998, 102, 6318-6322.

(36) Shah, K.; Chiu, P.; Jain, M.; Fortes, J.; Moudgil, B.; Sinnott, S. Langmuir 2005, 21, 5337-5342. 
(37) Shah, K.; Chiu, P.; Sinnott, S. B. J. Colloid Interface Sci. 2006, 296, 342-349.

(38) Dominguez, H. J. Phys. Chem. B 2007, 111, 4054-4059.

(39) Subramanian, S.; Wang, J. C. J. Chem. Phys. 2005, 123, 014706.

(40) Sholl, D. S. Langmuir 1998, 14, 862-867.

(41) Xu, Y.; Mavrikakis, M. J. Phys. Chem. B 2003, 107, 9298-9307.

(42) Berendsen, H. J. C.; van der Spoel, D.; van Drunen, R. Comput. Phys. Commun. 1995, 91, 43-56.

(43) Lindahl, E.; Hess, B.; van der Spoel, D. J. Mol. Model. 2001, 7, 306-317.

(44) van der Spoel, D.; Lindahl, E.; Hess, B.; Groenhof, G.; Mark, A. E.; Berendsen, H. J. C. J. Comput. Chem. 2005, 26, 1701-1718.

(45) Berger, O.; Edholm, O.; Jahnig, F. Biophys. J. 1997, 72, 20022013.

(46) Berendsen, H. J. C.; Postma, J. P. M.; van Gunsteren, W. F.; DiNola, A.; Haak, J. R. J. Chem. Phys. 1984, 81, 3684-3690.

(47) Hess, B.; Bekker, H.; Berendsen, H. J. C.; Fraaije, J. G. E. M. J. Comput. Chem. 1997, 18, 1463-1472.

(48) Miyamoto, S.; Kollman, P. A. J. Comput. Chem. 1992, 13, 952962.

(49) Essman, U.; Perela, L.; Berkowitz, M. L.; Darden, T.; Lee, H.; Pedersen, L. G. J. Chem. Phys. 1995, 103, 8577-8592.

(50) York, D. M.; Darden, T. A.; Pedersen, L. G. J. Chem. Phys. 1993, 99, 8345-8348.

(51) Patra, M.; Karttunen, M.; Hyvönen, M. T.; Lindqvist, P.; Falck, E.; Vattulainen, I. Biophys. J. 2003, 84, 3636-3645.
(52) Patra, M.; Karttunen, M.; Hyvönen, M. T.; Falck, E.; Vattulainen, I. J. Phys. Chem. B 2004, 108, 4485-4494.

(53) Berendsen, H. J. C.; Postma, J. P. M.; van Gunsteren, W. F.; Hermans, J. In Intermolecular Forces; Pullman, B., Ed.; Reidel: Dordrecht, The Netherlands, 1981; pp 331-342.

(54) Sammalkorpi, M.; Karttunen, M.; Haataja, M. J. Phys. Chem. B 2007, 111, 11722-11733.

(55) Humphrey, W.; Dalke, A.; Schulten, K. J. Mol. Graphics 1996, 14, 33-38.

(56) El-Barbary, A. A.; Telling, R. H.; Ewels, C. P.; Heggie, M. I.; Briddon, P. R. Phys. Rev. B 2003, 68, 144107.

(57) Telling, R.; Ewels, C.; El-Barbary, A.; Heggie, M. Nat. Mater. 2003, 2, 333-337.

(58) Lehtinen, P. O.; Foster, A. S.; Ma, Y.; Krasheninnikov, A. V.; Nieminen, R. M. Phys. Rev. Lett. 2004, 93, 187202.

(59) Kresse, G.; Furthmüller, J. Comput. Mater. Sci. 1996, 6, 15.

(60) Kresse, G.; Furthmüller, J. Phys. Rev. B 1996, 54, 11169.

(61) Throver, A.; Mayer, R. M. Phys. Status Solidi A 1978, 47, 11.

(62) Banerjee, S.; Hemraj-Benny, T.; Wong, S. Adv. Mater. 2005, 17, 17-29.

(63) Krasheninnikov, A. V.; Banhart, F. Nat. Mater. 2007, 6, 723-733.

(64) Hiura, H. Appl. Surf. Sci. 2004, 222, 372-381.

(65) Hiura, H. J. Mater. Res. 2001, 16, 1287-1292.

JP8043835 